\title{
Secuenciación del gen $C F T R$ en un grupo de pacientes chilenos con fibrosis quística
}

\author{
GUILLERMO LAY-SON R. ${ }^{1,2}$, MARCOS VÁSQUEZ D. ${ }^{1}$, ALONSO PUGA Y. ${ }^{3}$, \\ PATRICIO MANQUE M. ${ }^{3}$, GABRIELA REPETTO L. ${ }^{1,2}$ \\ 1. Centro de Genética Humana, Facultad de Medicina Clínica Alemana-Universidad del Desarrollo. \\ 2. Hospital Padre Hurtado. \\ 3. Centro de Genómica y Bioinformática, Universidad Mayor.
}

\section{ABSTRACT \\ CFTR gene sequencing in a group of Chilean patients with cystic fibrosis}

Introduction: Cystic fibrosis (CF) is an autosomal recessive genetic disorder caused by mutations of the CFTR gene, in which over 1,900 different mutations have been identified. In Chile, the diagnosis panel with the 36 most common mutations detects approximately $50 \%$ of all alleles, while for Caucasians, it is nearly $90 \%$. The objective of this study is to expand the capacity of mutational screening in Chilean patients and look for recurrent mutations at the national level. Method: The detection of unknown pathogenic alleles was assessed by CFTR gene sequencing in a selected group of patients from the National Cystic Fibrosis Foundation (NCFF). 39 patients, who met the CF diagnostic criteria and had only one allele identified according to the mutational panel, were studied. Massive sequencing was performed throughout the investigation and the main CFTR databases were used for analysis. Results: The second pathogenic allele was identified in 16 of 39 patients of this study (41\%), finding eleven different mutations that had not been reported in our population. We believe that the reason is that one of the variants had not been previously described. Conclusions: Mutations that had been described mainly in Hispanic and/or Mediterranean populations were identified. We found a variation that had not been previously reported, but not enough recurrent mutations that could explain the low rate of detection were found. Knowledge about mutations can provide appropriate genetic counseling and will be critical to evaluate the potential use of new targeted therapies for treating them.

(Key words: $C F T R$ gene, cystic fibrosis, Mutation, Massive sequencing).

Rev Chil Pediatr 2014; 85 (4): 448-454

\section{RESUMEN}

Introducción: La fibrosis quística (FQ) es un trastorno autosómico recesivo causado por mutaciones en el gen CFTR, en el cual se han identificado más de 1.900 mutaciones diferentes. En Chile, el panel diagnóstico

Recibido el 17 de julio de 2013, última versión aceptada para publicación el 27 de marzo de 2014.

Potenciales conflictos de interés: Este trabajo cumple con los requisitos sobre consentimiento /asentimiento informado, comité de ética, financiamiento, estudios animales y sobre la ausencia de conflictos de intereses según corresponda.

Financiamiento: Proyecto UDD 2012, No: 23400070

Correspondencia a:

Gabriela Repetto L.

E-mail: grepetto@udd.cl 
con las 36 mutaciones más comunes permite una tasa de detección cercana al $50 \%$ de los alelos, mientras que en caucásicos la tasa es casi de $90 \%$. El objetivo fue ampliar la capacidad de detección mutacional en los pacientes chilenos y buscar mutaciones que pudieran ser recurrentes a nivel local. Pacientes y Método: Se evaluó la detección de alelos patogénicos desconocidos mediante la secuenciación del gen CFTR en un grupo seleccionado de pacientes del Programa Nacional de FQ (PNFQ). Se analizaron 39 pacientes, que cumplían los criterios diagnósticos de FQ y que tenían sólo un alelo identificado con el panel mutacional. Se realizó secuenciación masiva y para el análisis se utilizaron las principales bases de datos de CFTR. Resultados: En este grupo seleccionado de pacientes se identificó el segundo alelo patogénico en 16 de los 39 pacientes (41\%), encontrándose once diferentes mutaciones que no se habían reportado en nuestra población. Según nuestro conocimiento, una de las variantes no había sido descrita previamente. Conclusiones: Se identificaron mutaciones que habían sido descritas principalmente en poblaciones hispánicas y/o mediterráneas. Encontramos una variante no reportada, aunque no encontramos mutaciones lo suficientemente recurrentes que pudieran explicar la baja tasa de detección. El conocimiento de las mutaciones permite otorgar un adecuado asesoramiento genético y será fundamental para evaluar el potencial uso de nuevas terapias específicas para las mutaciones. Palabras clave: Gen CFTR, Fibrosis quística, Mutación, Secuenciación masiva.

Rev Chil Pediatr 2014; 85 (4): 448-454

\section{Introducción}

La Fibrosis Quística (FQ) es una enfermedad multisistémica de herencia autosómica recesiva, con un incidencia de 1 en 2.000 a 1 en 3.000 nacimientos $^{1}$. En Chile, se estima que afecta a alrededor de 1 en 8.000 nacidos vi$\operatorname{vos}^{2,3}$.

El diagnóstico incluye al menos una característica clínica típica (enfermedad sinopulmonar crónica, insuficiencia pancreática, azoospermia obstructiva, síndrome perdedor de sal) más la evidencia de disfunción del canal de cloro CFTR (Cystic fibrosis transmembrane conductance regulator) demostrada a través de la detección de una elevada concentración de cloro en el sudor en 2 oportunidades, o una diferencia de potencial nasal transepitelial (NPD) característico de FQ o la detección de dos mutaciones en el gen CFTR causantes de $\mathrm{FQ}^{1,4}$.

Hasta la fecha, más de 1.900 mutaciones diferentes han sido identificadas en el gen $C F T R^{5}$, con frecuencias muy variables entre las distintas poblaciones. La p.F508 del (o deleción del aminoácido fenilalanina en la posición 508 del polipéptido) es la mutación más común, con una frecuencia global promedio de $66 \%$, pero con notables diferencias étnicas que van desde $28 \%$ en Asia a un $70 \%$ en los Europeos del norte ${ }^{6}$. Otras mutaciones llamadas "comunes" a nivel mundial, es decir con frecuencias $>1 \%$, incluyen p.G542X, p.G551D,
p.N1303K y p.W1282X. El resto son relativamente infrecuentes ${ }^{6,7}$, y para muchas de ellas, su rol patogénico no ha sido demostrado. Debido a que la gran mayoría de las mutaciones no tienen estudios funcionales, surgió el proyecto CFTR2 que ha permitido el análisis de más de 130 mutaciones sin información funcional previa ${ }^{8}$.

El panel mutacional para el diagnóstico molecular de FQ, que incluye las 36 mutaciones más comunes a nivel mundial, tiene una tasa de detección de menos de un 50\% de los alelos en nuestros pacientes ${ }^{9}$, mientras que en pacientes caucásicos con FQ este panel tiene una tasa de detección de alrededor de un $90 \%{ }^{10}$. Nuestro objetivo fue ampliar la capacidad de detección mutacional en los pacientes chilenos y buscar mutaciones que pudieran ser recurrentes a nivel local.

\section{Pacientes y Método}

\section{Pacientes y aprobaciones éticas}

Los pacientes que ingresan al Programa Nacional de Fibrosis Quística (PNFQ) cuentan con un diagnóstico clínico de la enfermedad con test anormal de electrolitos en sudor, según los criterios vigentes ${ }^{4}$. En ellos se realiza un estudio molecular, consistente en un panel que incluye las 36 mutaciones más comunes a nivel mundial. Para este reporte se estudia- 
ron treinta y nueve pacientes no emparentados, quienes permanecían con una sola mutación identificada con el panel de 36 mutaciones. Los participantes y sus representantes legales autorizaron y firmaron un consentimiento informado, que consideró la toma de muestra para el estudio molecular en búsqueda de mutaciones en el gen CFTR.

\section{Muestras}

Se estudió un grupo seleccionado de pacientes del Programa Nacional de Fibrosis Quística (PNFQ) en que faltaba detectar la segunda mutación patogénica, debido a que está reconocido que esta estrategia tiene más éxito en encontrar los alelos faltantes ${ }^{11}$. Mediante un método de secuenciación masiva, se analizó toda la secuencia codificante y los bordes exón-intrón del gen CFTR buscando mutaciones ya sea incluidas o no incluidas en el panel.

Se purificó ADN genómico desde linfocitos de sangre periférica utilizando el ensayo AxyPrep $^{\mathrm{TM}}$ Blood Genomic DNA Miniprep de Axygen Biosciences (Union City, California, EEUU).

\section{Análisis molecular}

Se prepararon las muestras utilizando el kit CFTR MASTR Assay v1.0 (Multiplicon N.V., Bélgica). El diseño experimental correspondió a la realización de dos PCR multiplex para cada paciente, generándose 34 amplicones específicos, que incluían los 27 exones del gen CFTR, más los segmentos intrónicos adyacentes.

La secuenciación masiva se realizó con el sistema de pirosecuenciación 454 GS Junior (Roche Diagnostics Corporation, Basilea, Suiza) ${ }^{12}$. Todos los procedimientos descritos se realizaron según las indicaciones de los fabricantes.

El resultado de las secuencias obtenidas para cada individuo fueron comparadas con la secuencia consenso de CFTR utilizando el software GS Amplicon Variant Analyser v2.5p1 (Roche Diagnostics Corporation, Basilea, Suiza) (figura 1). Todas las variantes detectadas se confirmaron con secuenciación capilar (método Sanger) y se contrastaron con los datos alojadas en las principales bases de datos de $\mathrm{FQ}^{5,8,13,14}$.

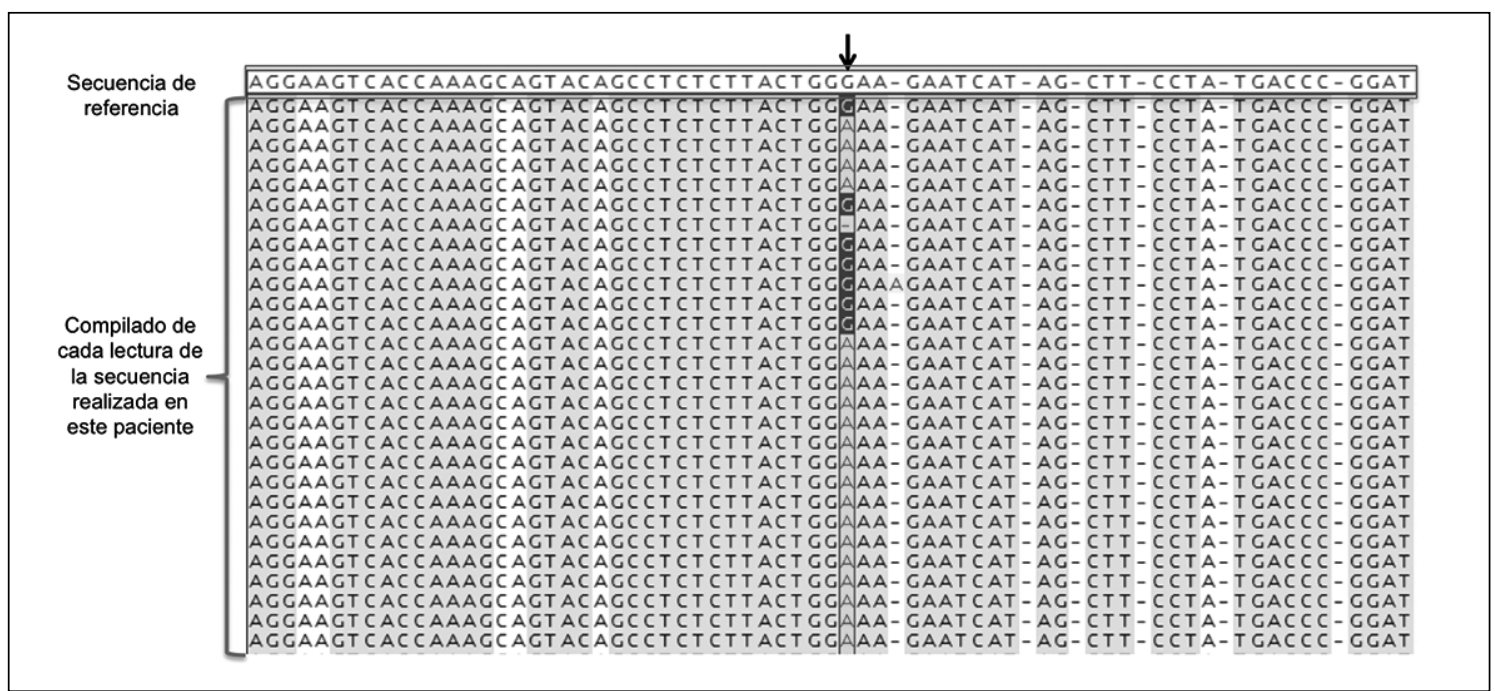

Figura 1. Detección de mutación c.308G $>$ A utilizando el software GS Amplicon Variant Analyzer (v2.5p1) de Roche. En la parte superior de la figura, la primera fila (en blanco) señala la secuencia de referencia del genoma_humano (versión hg19) para un segmento del exón 4 del gen CFTR. Desde la segunda fila hacia abajo corresponde al registro de cada lectura de esta secuencia que se obtuvo en este paciente. Se puede observar que en la posición del codón 308 (flecha superior), que según la secuencia de referencia corresponde normalmente al nucleótido G (primera fila), en algunas filas se leyó el nucleótido G (celdas sombreadas) y en otras el nucleótido A (celdas claras). Esto indica que el individuo tiene ambos alelos, es decir, es heterocigoto para esta variante. 
Tabla 1. Mutaciones detectadas por secuenciación masiva en cohorte de 39 pacientes chilenos con FQ portadores de un alelo desconocido

\begin{tabular}{|c|c|c|c|c|}
\hline $\begin{array}{l}\text { Mutación detectada } \\
\text { (nomenclatura actual*) }\end{array}$ & $\begin{array}{l}n \text { de } \\
\text { alelos }\end{array}$ & $\begin{array}{l}\text { Reporte en pacientes con FQ } \\
\text { ( } n^{\circ} \text { de alelos) }\end{array}$ & Efecto & $\begin{array}{l}\text { Denominación } \\
\text { antigua }\end{array}$ \\
\hline c.1330_1331delAT & 3 & Argentina $(1)^{a}$ & $\begin{array}{l}\text { Proteína truncada por genera- } \\
\text { ción de codón de término }\end{array}$ & 1460delAT \\
\hline c. $314 \mathrm{~T}>\mathrm{A}$ & 2 & Francia (1) ${ }^{\mathrm{a}}$ & $\begin{array}{l}\text { Cambio de aminoácido } \\
\text { Isoleucina por Asparagina }\end{array}$ & $1105 N$ \\
\hline c. $4046 \mathrm{G}>\mathrm{A}$ & 2 & Italia $(7)^{b, c}$, EEUU $(1)^{d}$ & $\begin{array}{l}\text { Cambio de aminoácido Glici- } \\
\text { na por Aspartato }\end{array}$ & G1349D \\
\hline c. $148 \mathrm{~T}>\mathrm{C}$ & 2 & España (2)e & $\begin{array}{l}\text { Cambio de aminoácido Serina } \\
\text { por Prolina }\end{array}$ & S50P \\
\hline c. $695 \mathrm{~T}>\mathrm{A}$ & 1 & $\begin{array}{l}\text { España (14)e,f, EEUU (hispanos) (5) })^{g, h} \\
\text { Francia (2) }{ }^{a} \text {, Brasil (1) }\end{array}$ & $\begin{array}{l}\text { Cambio de aminoácido Valina } \\
\text { por Aspartato }\end{array}$ & V232D \\
\hline c. $3266 \mathrm{G}>\mathrm{A}$ & 1 & $\begin{array}{l}\text { España (5)e, Brasil (2) })^{i, j} \text {, EEUU (hispa- } \\
\text { nos) }(2)^{g} \text {, Argentina }(1)^{k} \text {, Israel }(1)^{\prime}\end{array}$ & $\begin{array}{l}\text { Proteína truncada por genera- } \\
\text { ción de codón de término }\end{array}$ & W1089X \\
\hline c. $1647 \mathrm{~T}>\mathrm{G}$ & 1 & $\begin{array}{l}\text { Emiratos Árabes Unidos }(>30)^{m, n}, \\
\text { Colombia }(4)^{o} \text {, Israel }(4)^{p} \text {, Argelia }(2)^{p}, \\
\text { Marruecos }(2)^{q} \text {, Reino Unido }(2)^{p}, \\
\text { Portugal }(1)^{p} \text {, España }(1)^{p} \text {, Francia }(1)^{p}, \\
\text { Italia }(1)^{p}, \text { Brasil }(1)^{q} \text {, Argentina }(1)^{q}\end{array}$ & $\begin{array}{l}\text { Cambio de aminoácido Serina } \\
\text { por Arginina }\end{array}$ & $\mathrm{S} 549 \mathrm{R}(\mathrm{T}->\mathrm{G})$ \\
\hline c. $308 \mathrm{G}>\mathrm{A}$ & 1 & No descrita previamente & $\begin{array}{l}\text { Cambio de aminoácido } \\
\text { Glicina por Glutamato }\end{array}$ & G103E \\
\hline c. $1680-1 \mathrm{G}>\mathrm{A}$ & 1 & España $(1)^{r}$ & Alteración en splicing & $1812-1 \mathrm{G}->\mathrm{A}$ \\
\hline c. $1679+1 G>C$ & 1 & Francia $(2)^{s}$ Macedonia $(1)^{s}$ & Alteración en splicing & $1811+1 \mathrm{G}->\mathrm{C}$ \\
\hline c. $490-2 A>G$ & 1 & Argentina $(1)^{t}$ & Alteración en splicing & $622-2 A->G$ \\
\hline
\end{tabular}

FQ: Fibrosis quística. *nomenclatura estándar basada en las recomendaciones de la Human Genome Variation Society (HGVS) (den Dunnen JT and Antonarakis SE (2000). Hum. Mutat. 15: 7-12) a CFTR Mutation Database, http://www.genet.sickkids.on.ca/Home.html.

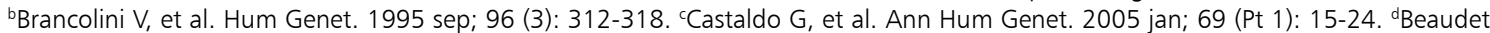
AL, et al. (1991) In: Tsui L-C, Romeo G, Greger R, Gorini S (eds) The identification of the CF (cystic fibrosis) gene-recent progress and new research strategies. Plenum Press, New York, London, pp 53-54. ${ }^{e}$ Alonso MJ, et al. Ann Hum Genet. 2007 mar; 71 (Pt 2): $194-201$. ${ }^{f}$ Casals T, et al. Hum Genet. 1997 dec; 101 (3): 365-70. 9Schrijver I, et al. J Mol Diagn. 2005 may; 7 (2): 289-99. hKammesheidt A, et al. Genet Med. 2006 sep; 8 (9): 557-62. 'Bernardino AL, et al. Genet Test. 2000; 4 (1): 69-74. 'Raskin S, et al. Genet Test. 2003 Fall; 7 (3): 213-8. 'Ramírez AM, et al. Mol Genet Metab. 2006 apr; 87 (4): 370-5. 'Shoshani T, et al. Hum Mol Genet. 1994 apr; 3 (4): $657-8$. mFrossard PM, et al. Eur Respir J. 1999 jan; 13 (1): 100-2. "Frossard PM, et al. Clin Genet 1999: 55: 496-497. 'Pérez MM, et al. J Cyst Fibros. 2007 may; 6 (3): 194-208. PEstivillX, et al. Hum Mutat. 1997; 10 (2): 135-54. 9Kerem E, et al. HumGenet. 1995 aug; 96 (2): 193-7. 'Chillón M, et al. Hum Mut 3: 223-230. 'Dujardin G, et al. J Cyst Fibros. 2011 may; 10 (3): 212-6. 'Oller de Ramírez AM, et al. Arch Argent Pediatr. 2008 aug; 106 (4): 310-9.

\section{Resultados}

Se analizaron 39 pacientes, que cumplían los criterios diagnósticos de FQ y que tenían sólo un alelo identificado con el panel mutacional. En este grupo seleccionado de pacientes se identificó el segundo alelo patogénico en 16 de los 39 pacientes (41\%), encontrándose once diferentes mutaciones, las cuales no se habían reportado en nuestra población. Los resultados se encuentran resumidos en la tabla 1. Cuatro de ellas fueron encontradas en más de un individuo. Al mismo tiempo, en cada paciente se corroboró la presencia de la otra mutación, previamente demostrada por el panel comercial.

Según nuestro conocimiento, la variante c.308G $>$ A (p.Gly103Glu), no ha sido descrita previamente en la literatura (figura $1 \mathrm{y}$ tabla 1).

Las mutaciones determinadas se clasificaron en: seis con sentido erróneo (missense), tres del sitio de corte y empalme (splicing), una mutación sin sentido (nonsense); y una deleción de 2 nucleótidos, que altera el marco de lectura (frameshift) y a la vez que genera un codón de termino prematuro. 
Adicionalmente, se encontró la variante $\mathrm{c} .3080 \mathrm{~T}>\mathrm{C}$ en un paciente con un alelo p.F508del. Esta variante se ha reportado como alelo complejo ya que comúnmente se encuentra en cis con la mutación p.F508del y por sí sola no tendría un rol patogénico ${ }^{8,15-21}$. Para corroborar que esta variante fue heredada en cis con la mutación p.F508del se deberán realizar estudios en los padres de este paciente.

\section{Discusión}

Este estudio comprendió un análisis completo de la secuencia codificante en un subgrupo de pacientes chilenos con FQ con sólo una mutación identificada. Se logró identificar un grupo de mutaciones que no están incluidas en el panel de las 36 mutaciones más comunes en la población mundial. La mayoría de estas mutaciones habían sido descritas en poblaciones con origen hispánico o del Mediterráneo. Esto es concordante con la observación de que las mutaciones más frecuentes en Chile tendrían un origen en la población del sur de Europa ${ }^{9}$.

En este grupo, no encontramos mutaciones lo suficientemente recurrentes que pudieran explicar en gran parte la razón de la baja tasa de detección en nuestro país, siendo probable que existan muchas mutaciones "privadas" con baja frecuencia. Es así como la mutación más frecuente encontrada en nuestro grupo de estudio, la mutación c.1330 1331delAT (p.Ile444X), fue identificada en tres pacientes diferentes. En el resto de los casos, tres mutaciones fueron encontradas en dos pacientes y ocho fueron detectadas sólo en un paciente. Las mutaciones están siendo analizadas en la cohorte completa de pacientes con FQ que tienen alelos sin identificar, para establecer su prevalencia en el total de pacientes del PNFQ.

Es interesante mencionar que la variante c.308G $>$ A (p.Gly103Glu), no ha sido descrita previamente, según la revisión de las distintas publicaciones y bases de datos de mutaciones de CFTR. Aunque no disponemos de datos funcionales, consideramos que es posiblemente patogénica ya que es una mutación que, cambia una glicina por un glutamato en el dominio de transmembrana MSD1 de CFTR. Es- tudios in vitro nos permitirán corroborar si el canal con la mutación es capaz de transportar iones en forma adecuada.

Basados en la experiencia internacional, con la estrategia de estudiar la secuencia codificante completa de CFTR y los segmentos intrónicos adyacentes a los exones, esperábamos acercarnos a un $90 \%$ de tasa de detección en el total de alelos ${ }^{4}$. Sin embargo, la gran proporción de alelos que permanecen no identificados plantean interrogantes sobre otras razones de esta baja tasa de detección. Un factor a considerar es referente a la certeza del diagnóstico clínico de FQ. Sin embargo, todos los pacientes que participaron de este análisis, al ingreso al PNFQ fueron seleccionados en base a criterios clínicos y de laboratorio compatible, por lo que problemas de selección de pacientes se hace menos probable.

Respecto a la metodología usada, la secuenciación masiva es una herramienta cada vez más accesible y confiable para ya sea para el estudio de mutaciones puntuales. Actualmente, es posible estudiar con fines clínicos los cambios genéticos que involucren una única base nucleotídica, en el conjunto completo de exones de un individuo (exoma) ${ }^{22}$, e incluso el genoma completo ${ }^{23}$. Debido a que se genera una gran cantidad de información, el gran desafío es la interpretación de la posible relevancia clínica de las variantes detectadas y que pueden corresponder a nuestra variabilidad "normal" entre individuos ${ }^{24}$.

En nuestro estudio, se hizo el análisis completo de la secuencia codificante del gen $C F T R$, no incluyendo regiones regulatorias ni la totalidad de la región intrónica, donde podrían encontrarse mutaciones con potencial patogénico. La secuenciación tiene limitaciones ya que no permite detectar rearreglos (deleciones y duplicaciones) del gen CFTR que también son causa de enfermedad, para ello será necesario analizar a estos pacientes con técnicas complementarias como el MLPA (Multiplex Ligation-dependent Probe Amplification), que detecta rearreglos cromosómicos de gran tamaño y no detectables por cualquier tipo de secuenciación ${ }^{25,26}$.

El análisis con metodologías complementarias (ej: MLPA) debe ser realizados en nuestros 
pacientes con FQ para poder obtener un diagnóstico molecular certero. Esta información redundará en un adecuado asesoramiento genético y en el último tiempo ha cobrado mayor relevancia, debido al advenimiento de terapias personalizadas según el tipo de mutación. A principios de 2012, la FDA ya ha aprobado el uso de un medicamento que es específico para la mutación p.G551D, el cual restaura la función proteica disminuyendo la concentración de cloruro en el sudor, y mejorando la función pulmonar $^{27,28}$, mientras que otros medicamentos están en ensayos clínicos avanzados ${ }^{29-31}$.

\section{Referencias}

1.- Welsh MJ, Ramsey BW, Accurso F, Cutting GR: Cystic Fibrosis. In: Scriver CR, Beaudet AL, Sly WS, et al., eds. The Metabolic and Molecular Bases of Inherited Disease. Vol 8th. New York: McGraw-Hill; 2001: 512188.

2.- Sánchez I, Pérez MA, Boza ML, et al: Consenso Nacional de Fibrosis Quística. Rev Chil Pediatr 2001; 72 (4): 356-80.

3.- Vega-Briceno LE, Guiraldes CE, Sánchez DI: [Cystic fibrosis: facing patient transition from the pediatrician to the internist]. Rev Med Chile 2006; 134 (3): 365-71.

4.- Moskowitz SM, Chmiel JF, Sternen DL, et al: Clinical practice and genetic counseling for cystic fibrosis and CFTR-related disorders. Genet Med 2008; 10 (12): 85168.

5.- Cystic Fibrosis Mutation Database (CFMDB) 2012. Available at: http://www.genet.sickkids.on.ca/cftr/StatisticsPage.html.

6.- Cystic Fibrosis Genetic Analysis Consortium: Population variation of common cystic fibrosis mutations. Hum Mutat 1994; 4 (3): 167-77.

7.- Bobadilla JL, Macek M, Fine JP, Farrell PM: Cystic fibrosis: a worldwide analysis of CFTR mutations-correlation with incidence data and application to screening. Hum Mutat 2002; 19 (6): 575-606.

8.- Cystic Fibrosis Foundation, Johns Hopkins University, The Hospital for Sick Children. Clinical and Functional Translation of CFTR (CFTR2) website. Available at: http://www.cftr2.org/index.php.

9.- Lay-Son G, Puga A, Astudillo P, Repetto GM: Cystic fibrosis in Chilean patients: Analysis of 36 common CFTR gene mutations. J Cyst Fibros 2011; 10 (1): 6670 .
10.- Watson MS, Cutting GR, Desnick RJ, et al: Cystic fibrosis population carrier screening: 2004 revision of American College of Medical Genetics mutation panel. Genet Med 2004; 6 (5): 387-91.

11.- Dequeker E, Stuhrmann M, Morris MA, et al: Best practice guidelines for molecular genetic diagnosis of cystic fibrosis and CFTR-related disorders-updated European recommendations. Eur J Hum Genet 2009; 17 (1): 5165.

12.- Voelkerding KV, Dames $S A$, Durtschi JD: Next-Generation Sequencing: From Basic Research to Diagnostics. Clinical Chemistry 2009; 55 (4): 641-58.

13.- The UMD-CFTR Knowledgebase. Available at: http:// www.umd.be/CFTR/.

14.- ABCMdb: The mutation browser. Available at: http:// abcmutations.hegelab.org/.

15.- Claustres M, Guittard C, Bozon D, et al: Spectrum of CFTR mutations in cystic fibrosis and in congenital absence of the vas deferens in France. Hum Mutat 2000; 16 (2): 143-56.

16.- Tzetis M, Efthymiadou A, Strofalis $S$, et al: CFTR gene mutations-including three novel nucleotide substitutions-and haplotype background in patients with asthma, disseminated bronchiectasis and chronic obstructive pulmonary disease. Hum Genet 2001; 108 (3): 216-21.

17.- Schrijver I, Ramalingam S, Sankaran R, et al: Diagnostic testing by CFTR gene mutation analysis in a large group of Hispanics: novel mutations and assessment of a population-specific mutation spectrum. J Mol Diagn 2005; 7 (2): 289-99.

18.- Kammesheidt A, Kharrazi M, Graham S, et al: Comprehensive genetic analysis of the cystic fibrosis transmembrane conductance regulator from dried blood specimens-implications for newborn screening. Genet Med 2006; 8 (9): 557-62.

19.- Alonso MJ, Heine-Suner D, Calvo M, et al: Spectrum of mutations in the CFTR gene in cystic fibrosis patients of Spanish ancestry. Ann Hum Genet 2007; 71 (Pt 2): 194-201.

20.- Fichou Y, Génin E, Le Maréchal C, Audrézet M-P, Scotet $V$, Férec $C$ : Estimating the age of CFTR mutations predominantly found in Brittany (Western France). Journal of Cystic Fibrosis 2008; 7 (2): 168-73.

21.- Dorfman R, Nalpathamkalam T, Taylor $C$, et al: Do common in silico tools predict the clinical consequences of amino-acid substitutions in the CFTR gene? Clin Genet 2010; 77 (5): 464-73.

22.- Need AC, Shashi V, Hitomi Y, et al: Clinical application of exome sequencing in undiagnosed genetic conditions. J Med Genet 2012: jmedgenet-2012-100819. 
23.- Worthey E, Bick D, Dimmock D, et al: Clinical diagnostic whole genome sequencing in a paediatric population: experience from our WGS genetics clinic. BMC Proceedings 2012; 6 (Suppl 6): O11.

24.- Gullapalli RR, Desai KV, Santana-Santos L, Kant $J A$, Becich $M J$ : Next generation sequencing in clinical medicine: Challenges and lessons for pathology and biomedical informatics. J Pathol Inform 2012; 3: 40 .

25.- Ferec C, Casals T, Chuzhanova N, et al: Gross genomic rearrangements involving deletions in the CFTR gene: characterization of six new events from a large cohort of hitherto unidentified cystic fibrosis chromosomes and meta-analysis of the underlying mechanisms. Eur J Hum Genet 2006; 14 (5): 567-76.

26.- Castellani C, Cuppens H, Macek M, et al: Consensus on the use and interpretation of cystic fibrosis mutation analysis in clinical practice. J Cyst Fibros 2008; 7 (3): 179-96.
27.- U.S. Food and Drug Administration. FDA approves Kalydeco to treat rare form of cystic fibrosis 2012. Available at: http://www.fda.gov/NewsEvents/Newsroom/ PressAnnouncements/ucm289633.htm.

28.- Davis PB, Yasothan U, Kirkpatrick P: Ivacaftor. Nature Reviews Drug Discovery 2012; 11 (5): 349-50.

29.- Clancy JP, Rowe SM, Accurso FJ, et al: Results of a phase IIa study of VX-809, an investigational CFTR corrector compound, in subjects with cystic fibrosis homozygous for the F508del-CFTR mutation. Thorax 2012; 67 (1): 12-18.

30.- Wilschanski M, Miller LL, Shoseyov D, et al: Chronic ataluren (PTC124) treatment of nonsense mutation cystic fibrosis. Eur Respir J 2011; 38 (1): 59-69.

31.- Knapp JM, Wood AB, Phuan P-W, et al: Structureactivity relationships of cyanoquinolines with correctorpotentiator activity in $\Delta \mathrm{F} 508$ cystic fibrosis transmembrane conductance regulator protein. J Med Chem 2012; 55 (3): 1242-51. 